\title{
Observer-based fault reconstruction for linear systems using adaptive sliding mode method
}

\author{
Hao Li ${ }^{1}$, Ying Yang1, Yifan Wei ${ }^{2}$, Fengzhi Dai ${ }^{2}$ \\ ${ }^{1}$ State Key Lab for Turbulence and Complex Systems, Department of Mechanics and Engineering Science, \\ College of Engineering, Peking University, \\ Beijing 100871, China \\ E-mail: li.hao@pku.edu.cn,yy@pku.edu.cn \\ ${ }^{2}$ College of Electronic Information and Automation, Tianjin University of Science and Technology, \\ Tianjin 300222, China \\ E-mail:weiyifan@tust.edu.cn,daifz@tust.edu.cn
}

\begin{abstract}
This paper studies the fault reconstruction problem for the continuous linear time-invariant system. A new disturbance-decoupled fault reconstruction design is proposed by cooperating with the idea of the adaptive sliding mode method. The new scheme does not impose restrictions on both the upper boundary of faults and the derivative of faults. Furthermore, the decoupling technique is used to deal with the disturbance input. Finally, simulation results are undertaken to validate the effectiveness of the proposed method.
\end{abstract}

Keywords: Fault reconstruction, adaptive sliding mode method, fault detection.

\section{Introduction}

In the last decade, automatic control systems have become more and more complex in order to meet the increasing demands for high production. This development calls for more system reliability, safety and dependability, which attracts compelling research interest on fields like state monitoring, fault diagnosis and fault tolerant control ${ }^{1}$. Furthermore, fault detection and isolation (FDI) plays an important role for timely detection of faults, especially in the field of active fault tolerant control.

Observer-based method is very fundamental tool to implement the fault reconstruction. The basic idea is to estimate the measured system outputs by using some kind of observer, then reconstruct the fault by utilizing the residual signal (error between the estimated output and the measured one). Many researchers focus on studies of sliding mode observer. The equivalent output injection concept based on sliding mode observer is used to reconstruct fault signal in Ref. 2. Ref. 3 proposes the FDI scheme with fault reconstruction capability for linear parameter varying system. Moreover, the adaptive method can also be used to design the fault reconstruction observer. A general framework in order to implement fast estimation is proposed in Ref. 4 by using the adaptive observer. In addition, fault reconstruction for nonlinear systems also attract many researchers' interest. The integrated design of observer-based fault detection for nonlinear system is addressed in Ref. 5. 
In this paper, our contribution is design of a fault reconstruction observer by using the idea of the adaptive sliding mode method. The condition that the upper boundary of faults should be known is not needed. Also, the method in this paper does not impose restrictions on the derivative of faults. Furthermore, the disturbance input is considered by using the decoupling technique.

The remainder of the paper is arranged as follows. In Section 2, the problem formulation is addressed. The novel disturbance-decoupled fault reconstruction design is studied in Section 3. Section 4 provides an example to illustrate the theoretical result. Finally, some concluding remarks are given in Section 5.

Through this paper, the notation is standard. $I_{N}$ and $\mathbb{R}^{n \times m}$ represent the identity matrix of dimension $N$ and the set of $n \times m$ real matrices, respectively. $\mathbb{R}^{n}$ denotes the set of real $n$-vectors ( $n \times 1$ matrices). The superscript $\mathrm{T}$ denotes transpose for real matrices.

\section{Preliminaries}

Consider the continuous linear time-invariant system:

$$
\begin{gathered}
\dot{x}(t)=A x(t)+B u(t)+E f(t)+M d(t) \\
y(t)=C x(t)
\end{gathered}
$$

where $x(t) \in \mathbb{R}^{n}$ is system state, $u(t) \in \mathbb{R}^{m}$ is system input, $y(t) \in \mathbb{R}^{p}$ is measured system output, $f(t) \in \mathbb{R}^{q}$ represents the time-varying actuator fault and $d(t) \in \mathbb{R}^{s}$ is the disturbance input and model uncertainties. It is assumed that $A, B, C, E$ and $M$ are known constant real matrices with appropriate dimensions. The pair ( $A, C$ ) is observable.

Remark.1 The actuator fault resulting from loss of effectiveness has been discussed in many researches. It can be regarded as the additive fault described in above system when the actuator is not totally damaged.

\section{A novel disturbance-decoupled fault reconstruction design}

In this section, we aim to design a fault reconstruction scheme such that the fault can be accurately estimated, even though there exists the external disturbance. The main idea is that designing a matrix decouples the system disturbance and utilizing residual information estimates the fault. The fault reconstruction observer for the faulty system is designed as

$$
\begin{gathered}
\dot{\hat{x}}(t)=A \hat{x}(t)+B u(t)+E \hat{f}(t)+L[y(t)-\hat{y}(t)] \\
\hat{y}(t)=C \hat{x}(t) \\
r(t)=T[y(t)-\hat{y}(t)]
\end{gathered}
$$

where $r(t) \in \mathbb{R}^{r}$ is the residual signal, $\hat{f}(t) \in \mathbb{R}^{q}$ represents the reconstruction of the actuator fault, $L \in \mathbb{R}^{n \times p}$ is observer gain matrix, and $T \in \mathbb{R}^{r \times p}$ is the matrix for decoupling the disturbance.

Denoting that state error $e(t)=x(t)-\hat{x}(t)$ and reconstruction error of the fault $\tilde{f}(t)=f(t)-\hat{f}(t)$, system error dynamics can be written as

$$
\begin{gathered}
\dot{e}(t)=(A-L C) e(t)+E \tilde{f}(t)+M d(t) \\
r(t)=T C e(t)
\end{gathered}
$$

In order to implement the disturbance-decoupled fault reconstruction scheme, the following assumption is given.

There exist a matrix $T$ and an observer gain matrix $L$ satisfying:

- $T C M=0$ and TCE has full column rank.

- The rows of $T C$ are left eigenvectors of Hurwitz $\bar{A}=A-L C$ corresponding to real eigenvalues.

Lemma 1. [Barbalat's Lemma] If a differentiable function $\varphi(t)$ satisfies $\varphi(t), \dot{\varphi}(t) \in L_{\infty}$, and $\varphi(t) \in L_{p}$ for some value of $p \in[1, \infty)$, then $\varphi(t) \rightarrow 0$ as $t \rightarrow 0$.

Theorem 1. Consider the fault system Eq. (1)-(2), the fault reconstruction observer Eq. (3)-(4) is realized by using:

$$
\begin{gathered}
\hat{f}(t)=\rho \frac{H r(t)}{\|H r(t)\|}, \\
\dot{\rho}=\alpha\|\operatorname{Hr}(t)\|,
\end{gathered}
$$

such that $\lim _{t \rightarrow \infty} r(t)=0$ and

$$
\lim _{(t \rightarrow \infty)} \int_{t}^{t+t_{s}} \tilde{f}(\tau) d \tau=0,
$$

where, $H=(P T C E)^{T}, \alpha$ is a design parameter, the matrix $\quad P>0 \quad$ satisfies $\Lambda^{T} P+P \Lambda=-Q<0$, $\Lambda=\operatorname{diag}\left\{\lambda_{1}, \lambda_{2}, \cdots, \lambda_{r}\right\}$, and $\lambda_{i}$ is the eigenvalue of $A-L C$ corresponding to the $i$ th row of $T C$.

Proof. We define $\tilde{\rho}=\rho-\rho^{*}$. The derivative of the residual $r(t)$ can be obtained as

$$
\begin{aligned}
\dot{r}(t) & =T C(A-L C) e(t)+T C E \tilde{f}(t) \\
& =\Lambda T C e(t)+T C E \tilde{f}(t) \\
& =\Lambda r(t)+T C E \tilde{f}(t)
\end{aligned}
$$

Consider a Lyapunov function candidate

$$
V(t)=\frac{1}{2} r(t)^{T} \operatorname{Pr}(t)+\frac{1}{2 \alpha} \tilde{\rho}^{2}
$$


Taking the time derivative of $V(t)$ along the trajectory of Eq. (11) yields

$$
\begin{aligned}
\dot{V}(t) & =\frac{1}{2} r(t)^{T}\left(\Lambda^{T} P+P \Lambda\right) r(t) \\
& +r(t)^{T} P T C E \tilde{f}(t)+\frac{1}{\alpha}\left(\rho-\rho^{*}\right) \dot{\rho} \\
& =-\frac{1}{2} r(t)^{T} Q r(t)+(H r(t))^{T} f(t)-(H r(t))^{T} \hat{f}(t) \\
& +\frac{1}{\alpha}\left(\rho-\rho^{*}\right) \dot{\rho} \\
& =-\frac{1}{2} r(t)^{T} Q r(t)+(H r(t))^{T} f(t)-(H r(t))^{T} \hat{f}(t) \\
& +\left(\rho-\rho^{*}\right)\|H r(t)\| \\
& \leqslant-\lambda_{\min }(Q)\|r(t)\|^{2}+\|H r(t)\|\|f(t)\| \\
& -(H r(t))^{T} \hat{f}(t)+\left(\rho-\rho^{*}\right)\|H r(t)\| \\
& \leqslant-\lambda_{\min }(Q)\|r(t)\|^{2}+\|H r(t)\|\left(\|f(t)\|-\rho^{*}\right) \\
& -(H r(t))^{T} \hat{f}(t)+\rho\|H r(t)\|
\end{aligned}
$$

If we choose a large enough $\rho^{*}$, then $\|f(t)\|-\rho^{*} \leq 0$. The above inequality can be written as

$$
\begin{aligned}
\dot{V}(t) \leqslant-\lambda_{\text {min }}(Q)\|r(t)\|^{2}-(H r(t))^{T} \hat{f}(t) & +\rho\|H r(t)\|
\end{aligned}
$$

Based on Eq.(8), we can obtain

$$
\dot{V}(t) \leqslant-\lambda_{\text {min }}(Q)\|r(t)\|^{2}
$$

Hence, $V(t) \in L_{\infty}$ (i.e., $V(t)$ is uniformly bounded), which implies $r(t), \rho^{*} \in L_{\infty}$. Then $\rho=\tilde{\rho}+\rho^{*}$ is uniformly bounded. Since $\lim V(t)=0$, we obtain that $r(t) \in L_{2}$ by integrating both sides of Eq. (15) from 0 to $\infty$. Using Lemma 1 , we conclude that $\lim _{t \rightarrow \infty} r(t)=0$.

Since $\lim r(t)=0$, it holds that

$$
\begin{gathered}
\lim _{t \rightarrow \infty} \int_{t}^{t+t_{s}} \Lambda r(\tau) d \tau=0 \\
\lim _{t \rightarrow \infty} \int_{t}^{t+t_{S}} \operatorname{TCE\tilde {f}}(\tau) d \tau \\
=\lim _{(t \rightarrow \infty)}\left[r\left(t+t_{s}\right)-r(t)\right] \\
=0
\end{gathered}
$$

Utilizing the condition that $T C E$ is full column rank, it follows that

$$
\lim _{t \rightarrow \infty} \int_{t}^{t+t_{s}} \tilde{f}(\tau) d \tau=0
$$

This ends the proof.

Remark 2. By cooperating with the adaptive sliding mode idea, the upper bound of the fault information is not necessary. Compared to Ref. 6, the condition that the fault has the known upper bound (i.e. $\|f(t)\| \leq f_{\text {upper }}$, where $f_{\text {upper }}$ is a prior) can be released.

Remark 3. Note that the discontinuous signal is used to design the fault reconstruction observer. In the practical problem, the boundary layer methodology can be taken into account to deal with the chattering trouble.

\section{Simulation Results}

In this section, a simulation example on a vertical takeoff and landing (VTOL) linear aircraft model is given to validate the effectiveness of the proposed fault reconstruction approach.

The linearization dynamics of the aircraft can be described by system (1)-(2), where matrix coefficients have following values ${ }^{6}$

$$
\begin{aligned}
& A=\left[\begin{array}{cccc}
-0.0366 & 0.0271 & 0.0188 & -0.4555 \\
0.0482 & -1.01 & 0.0024 & -4.0208 \\
0.1002 & 0.3681 & -0.707 & 1.420 \\
0.0 & 0.0 & 1.0 & 0.0
\end{array}\right], \\
& B=\left[\begin{array}{cc}
0.4422 & 0.1761 \\
3.5446 & -7.5922 \\
-5.52 & 4.49 \\
0.0 & 0.0
\end{array}\right], \quad C=\left[\begin{array}{llll}
1 & 0 & 0 & 0 \\
0 & 1 & 0 & 0 \\
0 & 0 & 1 & 0 \\
0 & 0 & 0 & 1
\end{array}\right] \text {, } \\
& M=\left[\begin{array}{llll}
0.4 & 0 & 0 & 0.4
\end{array}\right]^{T} \text {. }
\end{aligned}
$$

In this example, we consider the actuator fault so $E=B$ holds.

The decoupled matrix $T$ in Eq.(5) can be designed as

$$
T=\left[\begin{array}{llll}
0 & 1 & 0 & 0 \\
0 & 0 & 1 & 0
\end{array}\right],
$$

it is easy to verify that $T C M=0$ and $\operatorname{rank}(T C E)=2$. The observer gain $L$ in Eq.(3) can be designed by pole assignment method to place eigenvalues of $A-L C$ into locations $\left[\begin{array}{llll}-3 & -2 & -7 & -3\end{array}\right]$.

$$
L=\left[\begin{array}{cccc}
4.9634 & 0.0271 & 0.0188 & -0.4555 \\
0.0482 & 4.9900 & 0.0024 & -4.0208 \\
0.1002 & 0.3681 & 5.2930 & 1.4200 \\
0 & 0 & 1.0000 & 4.0000
\end{array}\right] .
$$

We choose the design parameter $\alpha=1$. The design matrix parameter $P$ can be solved by applying the linear matrix inequality toolbox in MATLAB. We can obtain

$$
H=\left[\begin{array}{cc}
12.5801 & -43.0987 \\
-26.9453 & 35.0567
\end{array}\right] \text {. }
$$


We randomly choose initial states of the system. The disturbance signal follows Gaussian distribution $d \sim N(0,0.2)$. It is assumed that the fault signal $f(t)$ has the following form:

$$
\begin{gathered}
f_{1}(t)=0, \\
f_{2}(t)= \begin{cases}0, & 0 \leq t<2 s \\
0.02 \sin t+0.03, & 2 s \leq t \leq 20 s .\end{cases}
\end{gathered}
$$
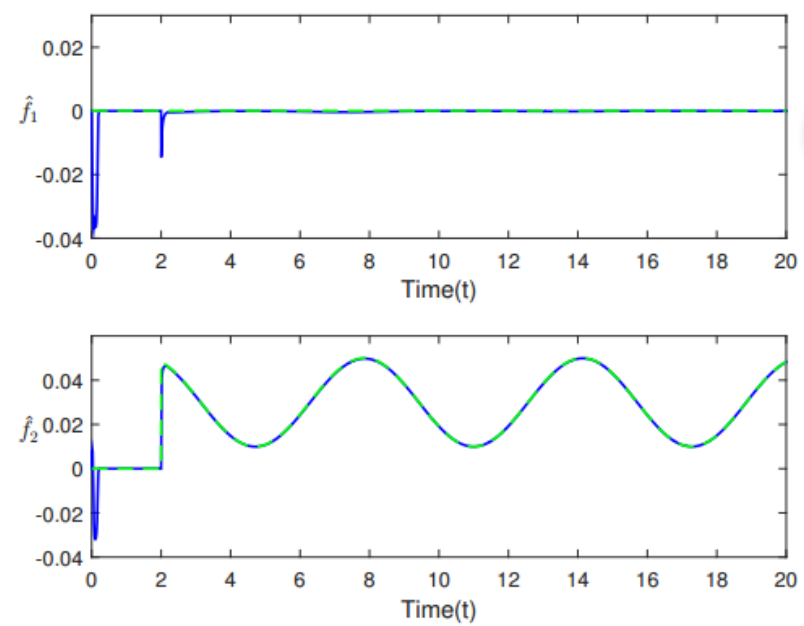

Fig. 1. Fault $f(t)$ (dotted line) and fault reconstruction $\hat{f}(t)$ (solid line).
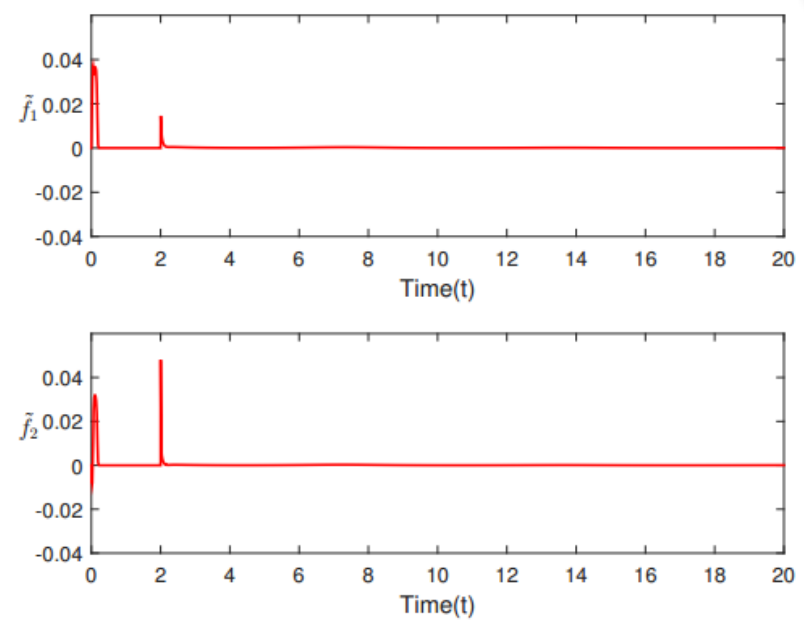

Fig. 2. Reconstruction error of fault $\tilde{f}(t)$.

Note that the proposed fault reconstruction method can effectively estimate the actual additive fault. Fig.1 shows the performance of the proposed scheme. As expected, the fault reconstruction can accurately estimate the actual fault. Fig. 2 shows the reconstruction error $\tilde{f}(t)$, which can effectively validate the previous theoretical result.

\section{Conclusion}

The fault reconstruction problem for the continuous linear time-invariant system is studied in this paper. A new disturbance-decoupled fault reconstruction design is proposed. Conditions that both the upper boundary of faults and the derivative of faults are released. In addition, the decoupling technique is used to deal with the disturbance input.

\section{Acknowledgments}

This work is supported by the National Basic Research Program of China (973 program) 2012CB821202 and by the National Natural Science Foundation of China under grant 61174052 .

\section{References}

1. S. X. Ding, Data-driven design of fault diagnosis and fault-tolerant control systems, (Springer, 2014).

2. C. Edwards, S. K. Spurgeon, and R. J. Patton, Sliding mode observers for fault detection and isolation, Automatica, 36 (4) (2000) 541-553.

3. H. Alwi and C. Edwards, Robust fault reconstruction for linear parameter varying systems using sliding mode observers, International Journal of Robust and Nonlinear Control, 24 (14) (2014) 1947-1968.

4. K. Zhang, B. Jiang, V. Cocquempot et al., Adaptive observer-based fast fault estimation, International Journal of Control Automation and Systems, 6 (3) (2008) 320-326.

5. Y. Yang, S. X. Ding, and L. Li, On observer-based fault detection for nonlinear systems, Systems \& Control Letters, 82 (2015) 18-25.

6. L. Cui, H. Liu, and Y. Yang, Youla parameterization fault-tolerant controller design for aircraft, Journal of Guidance, Control, and Dynamics, 36 (5) (2013) 1533-1538. 